\title{
ORIGINAL ARTICLE Impact of antiretroviral therapy on pregnancy outcomes
}

\author{
C D Aniji, ${ }^{1}$ FCOG (SA); O A Towobola, ${ }^{1} \mathrm{PhD} ;$ M E Hoque, ${ }^{2}$ MSc; T J Mashamba, ${ }^{1}$ MB ChB; S Monokoane, ${ }^{1}$ FCOG $($ SA $)$ \\ ${ }^{1}$ Department of Obstetrics and Gynaecology, University of Limpopo, Medunsa Campus, Pretoria, South Africa \\ ${ }^{2}$ Graduate School of Business and Leadership, University of KwaZulu-Natal, Westville Campus, Durban, South Africa
}

Corresponding author: M E Hoque (muhammad.ehsanul@gmail.com)

Background. The majority of HIV-positive women in South Africa are of reproductive age, and pregnancies among women using antiretroviral therapy (ART) are common. However, there are mixed data regarding the impact of ART on pregnancy outcomes.

Objective. To examine the impact of ART on pregnancy outcome according to the timing of initiation of treatment.

Methods. A retrospective cohort study was conducted among women delivering at a tertiary hospital from 1 October 2008 to 31 March 2009.

Results. A total of 245 mothers were receiving ART: 76 mothers (31\%) started ART pre-conception and 169 mothers (69\%) started ART after the first trimester. No significant differences were observed in the rates of preterm delivery and low birth weight (LBW) between the pre- and post-conception groups ( $21 \%$ v. $24 \%$ and $21 \%$ v. $25 \%$, respectively).

Conclusion. In this cohort of women receiving ART in pregnancy, timing of ART initiation did not have any adverse effect on the measured pregnancy outcomes such as preterm delivery and LBW.

S Afr J HIV Med 2013;14(4):176-178. DOI:10.7196/SAJHIVMED.834

口iritiv

South Africa (SA), like many countries in sub-Saharan Africa, has a high HIV disease burden. According to 2008 reports by the World Health Organization (WHO) and the Joint United Nations Programme on HIV and AIDS (UNAIDS), an estimated 6 million adults and children were living with HIV in SA by the end of 2007, including approximately 3.2 million (54\%) women aged $\geq 15$ years. ${ }^{[1,2]}$ This has a significant impact on vertical transmission and maternal mortality. The Fourth Report on Confidential Enquiries into Maternal Deaths in South Africa 2005 - 2007 showed that pregnancy-unrelated infections, mostly HIV/AIDS, were responsible for $43.7 \%$ of all reported maternal deaths. ${ }^{[3-5]}$

Varied results have been reported on the risk of adverse pregnancy outcomes in HIV-positive women treated with antiretroviral therapy (ART). Several studies from Europe have suggested that the initiation of ART during pregnancy may be associated with an increased risk of preterm delivery, particularly with protease inhibitor (PI)-containing regimens. ${ }^{[6-8]}$ However, these findings have been contradicted by studies from Brazil and North America showing no association between maternal ART use and pregnancy outcomes. ${ }^{[-12]}$

In Africa, there have been some studies that evaluated the impact of ART on pregnancy outcomes. In a Cote d'Ivoire study ${ }^{[13]}$ it was highlighted that ART in pregnant women with advanced HIV disease substantially reduced mother-to-child transmission, but was associated with low birth weight (LBW). A matched case-control study conducted in Nigeria ${ }^{[14]}$ found that HIV-positive women were significantly more likely to have intrauterine growth restriction, preterm labour and LBW babies than HIV-negative women.

Given the large number of HIV-positive pregnant women in SA, and the importance of ART use for promoting maternal and child health in 
Table 1. Comparison of maternal age, parity and mode of delivery between the pre- and post-conception ART groups

\begin{tabular}{|c|c|c|c|}
\hline Variable & Pre-conception $(N=76)$ & Post-conception $(N=169)$ & $p$-value \\
\hline Age, mean $( \pm S D)$ & $31.4( \pm 5.3)$ & $29.2( \pm 5.4)$ & 0.003 \\
\hline \multicolumn{4}{|l|}{ Parity, $n(\%)$} \\
\hline 0 & $4(5.3)$ & $31(18.3)$ & 0.003 \\
\hline $1-2$ & $49(64.5)$ & $118(69.8)$ & 0.378 \\
\hline$\geq 3$ & $23(30.2)$ & $20(11.8)$ & 0.047 \\
\hline Mode of delivery, $n(\%)$ & & & 0.568 \\
\hline Vaginal & $52(68.4)$ & $113(66.9)$ & \\
\hline Caesarean section & $24(31.6)$ & $56(33.1)$ & \\
\hline
\end{tabular}

Table 2. Comparison of gestational age and birth weight between the pre- and post-conception ART groups

\begin{tabular}{|c|c|c|c|}
\hline Variable & Pre-conception $(N=76)$ & Post-conception $(N=169)$ & $p$-value \\
\hline Gestational age, $n(\%)$ & & & 0.348 \\
\hline Pre-term & $16(21.1)$ & $41(24.3)$ & \\
\hline Term & $60(78.9)$ & $128(75.7)$ & \\
\hline Fetal weight $(\mathrm{g}),{ }^{*} n(\%)$ & & & 0.945 \\
\hline$\geq 2500$ & $60(78.9)$ & $128(75.7)$ & \\
\hline $1500-2499$ & $13(17.1)$ & $35(20.7)$ & \\
\hline $1000-1499$ & $2(2.6)$ & $4(2.4)$ & \\
\hline $500-999$ & $1(1.3)$ & $2(1.2)$ & \\
\hline \multicolumn{4}{|l|}{ ART $=$ antiretroviral therapy. } \\
\hline 'Fisher's exact test. & & & \\
\hline
\end{tabular}

this population, it is pertinent to investigate any effects that ART may have on pregnancy outcomes.

The aim of this study was to investigate the impact of ART on pregnancy outcome, according to the timing of initiation of treatment, in a cohort of HIV-positive pregnant women at Dr George Mukhari Hospital (DGMH) in Ga-Rankuwa, SA.

\section{Methods}

We performed a retrospective medical record review of HIV-positive pregnant women who delivered at DGMH from 1 October 2008 to 31 March 2009. DGMH has a large academic obstetric unit with approximately 8500 deliveries per annum. The study included HIVpositive pregnant women who booked at the antenatal clinic at DGMH and had either been receiving ART before pregnancy (pre-conception ART group) or started ART after the first trimester of pregnancy (postconception ART group). Women with multiple pregnancies and those who defaulted ART and/or antenatal clinic visits were excluded from the analysis.

Data on demographic and obstetric characteristics and ART use were collected from the labour ward delivery register, patients' antenatal cards and/or hospital records. Data were analysed using SPSS (version 17.0). Term delivery was defined as birth at a gestational age of $\geq 37$ weeks, where ultrasound was used to date the pregnancy before 20 weeks' gestation; or a birth weight of $\geq 2500 \mathrm{~g}$ in cases where pregnancy was not dated with ultrasound before 20 weeks' gestation. All statistical tests were performed using two-sided tests at the $p \leq 0.05$ level of significance.

Ethical approval for the study was obtained from the Medunsa Research and Ethics Committee of the University of Limpopo.

\section{Results}

A total of 245 mothers were receiving ART during the study period; 76 (31\%) were receiving ART pre-conception and 169 mothers (69\%) started ART post conception after the first trimester. The average age of the participants was 31 and 29 years in the pre- and post-conception groups, respectively $(p=0.003)$. Significantly more women in the postconception group were primigravidae than in the pre-conception group $(18 \%$ v. $5 \%$, respectively; $p=0.003)$. The rate of caesarean sections was similar in the pre- and post-conception groups (Table 1).

In the pre-conception group, 73 women were on a regimen of two nucleoside reverse transcriptase inhibitors (NRTIs) with a nonnucleoside reverse transcriptase inhibitor (NNRTI), while 3 women were on PI-containing regimens. The duration of treatment prior to conception ranged from 4 to 60 months. All the women in this group were in relatively good health, with sustained viral suppression prior to conception. In the post-conception group, 161 women were using two NRTIs with an NNRTI, while 8 women were on PI-containing regimens. All women in this group had $\mathrm{CD}^{+}$counts $<200$ cells $/ \mu$ l per the national ART guidelines at the time of the study. The duration of treatment before delivery ranged from 2 to 22 weeks.

Table 2 compares pregnancy outcomes between the two groups. Results indicated that preterm delivery rates were similar between the pre- and post-conception groups $(p=0.348)$. With regards to LBW, the rate was slightly lower $(21 \%)$ in the pre-conception group than in the postconception group (24\%), but the difference was not significant ( $p=0.945)$.

\section{Discussion}

We investigated the effect of ART on pregnancy outcomes according to the timing of initiation of treatment. Accordingly, we did not find any 
negative impact of this therapy on pregnancy outcomes, regardless of the timing of treatment initiation.

Those women who were receiving ART prior to conception, irrespective of the duration of treatment, had a similar rate of preterm delivery compared with those who commenced ART after the first trimester of pregnancy. A study conducted in the $\mathrm{USA}^{[10]}$ also reported a non-significant difference in the rate of preterm delivery between pre- and post-conception ART groups. It would seem that in this study population, the use of antiretrovirals did not have any effect, whether positive or negative, on the rate of preterm deliveries. It is important to point out that the use of illicit drugs, alcohol abuse and cigarette smoking - all important causes of preterm labour - were negligible in this setting.

However, our results are in contrast to the findings of some other studies conducted in Europe, Cote d'Ivore and Nigeria, which concluded that patients who were receiving ART prior to conception had an increased risk of preterm delivery compared with those who commenced ART post conception. ${ }^{[8,12-14]}$

With regards to LBW in our study, the rates were similar: $17.1 \%$ and $20.7 \%$ in the pre- and post-conception groups, respectively. This result is in line with other studies conducted in Brazil and the USA, ${ }^{[11,15]}$ both of which did not find any association between birth weight and the use of ART. However, contrasting results from Europe, Cote d'Ivore and Nigeria indicated higher rates of LBW among women receiving ART. ${ }^{[8,13,14]}$

\section{Study limitations}

This was a retrospective study that focused on women delivering live infants in health facilities and did not account for early pregnancy loss or home deliveries, thus constituting an important potential bias. Several other factors with known influence on pregnancy outcome including other infectious diseases, socioeconomic information of the mothers (e.g. educational level and employment status) were not measured and could not be evaluated in this study.

\section{Conclusion}

In summary, the results of this study suggest that ART initiated before or during pregnancy does not have any adverse effect on measured pregnancy outcomes such as preterm delivery and LBW.
Acknowledgement. We thank all members of staff in the labour ward for their efforts in entering data into the maternity records, and the clerks in the records department who assisted with patient file retrieval.

\section{References}

1. UNAIDS/WHO Epidemiological Fact Sheets on HIV and AIDS, 2008 Update. http://apps.who.int/globalatlas/predefined Reports/EFS2008 (accessed 28 March 2011).

2. National Department of Health. National Antenatal Sentinel HIV and Syphilis prevalence survey in South Africa 2009. http://www.doh.za/docs (accessed 28 March 2011).

3. National Department of Health. Saving Mothers. Fourth Report on Confidential Enquiries into Maternal Deaths in South Africa 2005 - 2007. http://www.doh.za/ docs/reports (accessed on 28 March 2011).

4. National Department of Health. National Antiretroviral Treatment Guidelines. http://www.doh.za/docs/reports (accessed 23 March 2011).

5. National Department of Health. Clinical Guidelines for the management of HIV and AIDS in adults and adolescents. http://www.doh.za/docs/reports (accessed 17 January 2011).

6. Martin F, Taylor GP. Increased rates of preterm delivery are associated with the initiation of highly active antiretroviral therapy in pregnancy: A single centre cohort study. J Infect Dis 2007;196(4):558-561. [http://dx.doi.org/10.1086/519848]

7. Grosch-Woerner I, Puch K, Maier RF, et al. Increased rate of prematurity associated with antenatal antiretroviral treatment in German/Austrian cohort of HIV-1 infected women. HIV Med 2008;9:6-13. [http://dx.doi.org/10.1111/j.1468-1293.2008.00520.x]

8. Thorne C, Patel D, Newell ML. Increased risk of adverse pregnancy outcomes in HIVinfected women treated with highly active antiretroviral therapy in Europe. AIDS 2004;18(17):2337-2379.

9. Carceller A, Ferreira E, Alloul S, Lapointe N. Lack of effect on prematurity, birth weight and infant growth from exposure to protease inhibitors in utero and after birth. Pharmacotherapy 2009;29(11):1289-1296. [http://dx.doi.org/10.1592/ phco.29.11.1289]

10. Patel K, Shapiro DE, Brogly SB, Livingston EG, Stek AM, Bardeguez AD. Prenatal protease inhibitor use and risk of preterm birth among HIV-infected women initiating antiretroviral drugs during pregnancy. J Infect Dis 2010;201(7):1035-1044. [http://dx.doi.org/10.1086/651232]

11. Szyld EG, Warley EM, Freimanis L, et al. Maternal antiretroviral drugs during pregnancy and infant low birth weight and preterm birth. AIDS 2006;20(18):23452353. [http://dx.doi.org/10.1097/01.aids.0000253362.01696.9d]

12. Machado ES, Hofer CB, Costa TT, et al. Pregnancy outcome in women infected with HIV-1 receiving combination antiretroviral therapy before versus after conception. Sex Transm Infect 2009;85(2):82-87. [http://dx.doi.org/10.1136/sti.2008.032300]

13. Ekouevia DK, Coffiea PA, Becquet R, et al. HAART, antiretroviral therapy in pregnant women with advanced HIV disease and pregnancy outcomes in Abidjan, Cote d'Ivoire. AIDS 2008;22(14):1815-1820.

14. Olagbuji BN, Ezeanochie MC, Ande AB, Oboro VO. Obstetric and perinatal outcome in HIV positive women receiving HAART in urban Nigeria. Arch Gynecol Obstec 2010;281(6):991-994. [http://dx.doi.org/10.1007/s00404-009-1186-x]

15. Schulte J, Dominguez K, Sukalac T, Bohannon B, Fowler MG. Declines in low birth weight and preterm birth among infants who were born to HIV-infected women during an era of increased use of maternal antiretroviral drugs: Pediatric Spectrum of HIV Disease, 1989-2004. Pediatrics 2007;119(4):e900-e906. [http://dx.doi. org/10.1542/peds.2006-1123 